\title{
BMJ Open Protocol of the randomised placebo controlled pilot trial of the management of acute sciatica (SCIATICA): a feasibility study
}

\author{
Marissa Nichole Lassere, ${ }^{1}$ Kent Robert Johnson, ${ }^{2}$ Jeanette Thom, ${ }^{3}$ Grant Pickard, ${ }^{4}$ \\ Peter Smerdely ${ }^{5}$
}

To cite: Lassere MN, Johnson KR, Thom J, et al. Protocol of the randomised placebo controlled pilot trial of the management of acute sciatica (SCIATICA): a feasibility study. BMJ Open 2018;8:e020435. doi:10.1136/ bmjopen-2017-020435

- Prepublication history for this paper is available online. To view these files, please visit the journal online (http://dx.doi. org/10.1136/bmjopen-2017020435).

Received 3 November 2017 Revised 3 May 2018 Accepted 11 May 2018

Check for updates

${ }^{1}$ Rheumatology Department, St George Hospital, Kogarah, New South Wales, Australia

${ }^{2}$ St George Clinical School, University of New South Wales, Kogarah, New South Wales, Australia

${ }^{3}$ School of Medical Sciences, University of New South Wales, Kensington, New South Wales, Australia

${ }^{4}$ Medical Assessment Unit, St George Hospital, Kogarah, New South Wales, Australia

${ }^{5}$ Department of Aged Care, St

George Hospital, Kogarah, New South Wales, Australia

Correspondence to

Professor Marissa

Nichole Lassere;

m.lassere@unsw.edu.au

\section{ABSTRACT}

Introduction Acute sciatica (symptom duration less than 4 weeks), a major cause of pain and disability, is a common presentation to medical practices and hospital emergency departments. Selective CT fluoroscopy transforaminal epidural steroid injection is often used with the hope of reducing pain and improving function. Recently, there has been interest in using systemic corticosteroids in acute sciatica. However, there is limited evidence to inform management of selective CT fluoroscopy transforaminal epidural steroid in subacute and chronic sciatica and there is no evidence in acute sciatica, even though the practice is widespread. There is also limited evidence for the use of systemic corticosteroids in acute sciatica. Furthermore, the management of selective CT fluoroscopy transforaminal epidural steroid versus systemic steroids has never been directly studied.

Methods and analysis SCIATICA is a pilot/feasibility study of patients with acute sciatica designed to evaluate the feasibility of undertaking a blinded four-arm randomised controlled intervention study of (1) selective CT fluoroscopy transforaminal epidural steroid (arm 1), (2) selective CT fluoroscopy transforaminal epidural saline (arm 2), (3) 15 days tapering dose of oral steroids (arm 3) and (4) a sham epidural and oral placebo control (arm 4). This feasibility study is designed to evaluate head-to-head, route versus pharmacology of interventions. The primary outcome measure is the Oswestry Disability Index (ODI) at 3 weeks. Secondary outcome is the $\mathrm{ODI}$ at 48 weeks. Other outcomes include numerical rating scale for leg pain, Pain DETECT Questionnaire, quality of life, medication use, rescue procedures or surgery, and adverse events. Results of outcomes from this randomised controlled trial will be used to determine the feasibility, sample size and power calculations for a large multicentre study.

Ethics and dissemination The study has been approved by South Eastern Sydney Local Health District Human Research Ethics Committee (HREC/15/331/POHW/586). Trial registration number NCT03240783; Pre-results.

\section{INTRODUCTION}

The colloquial definition of sciatica is pain in the buttock and leg, and it is a term understood by the non-professional population.
Strengths and limitation of this study

- In the setting of acute sciatica (less than 4 weeks duration), this four-arm trial evaluates the feasibility of undertaking a head-to-head route versus pharmacology of intervention randomised controlled trial (RCT) by comparing epidural steroid with systemic steroids, and epidural steroid with epidural saline, and includes blinding with both oral placebo and sham injection across each arm. Such a trial directly provides risk versus benefit of interventions of interest.

- Evaluates feasibility of recruiting and protocol adherence of participants from different referral and demographic settings: public hospital inpatients, private hospital inpatients, emergency department presentations and general practitioner visits.

- Evaluates the challenge of recruiting participants to a RCT of acute sciatica where there often is an expectation of treatment benefit of a procedural intervention by healthcare professionals (and patients given frequent use of the internet for healthcare advice), because of a large placebo effect, the natural history of the condition and extrapolation of results from case series or RCTs with different inclusion criteria, but where there is no direct RCT evidence of benefit and risk.

The anatomical pathology is usually caused by lumbosacral disc herniation and degenerative lumbosacral spondylosis involving the $\mathrm{L} 2 / 3$ to $\mathrm{L} 5 / \mathrm{S} 1$ intervertebral discs and foramina. ${ }^{1}$ Therefore, sciatica can be associated with numbness, paraesthesia and weakness in the leg. The terms radicular pain and radiculopathy describe this neurological component of the pathology by healthcare professionals and researchers. ${ }^{2}$ Radicular pain is thought to arise from ectopic activation of nociceptive afferent fibres in a spinal nerve or its roots from ischaemia or inflammation. ${ }^{3}$ Radiculopathy indicates that there is conduction block of the spinal nerve or its 
roots from either mechanical compression or ischaemia. Nonetheless, the terms are still used interchangeably and inconsistently in the randomised controlled trial (RCT) literature. ${ }^{45}$ This study defines the term sciatica as radicular pain with or without radiculopathy from lumbosacral nerve root pathology. The definition of acute sciatica in the RCT and systematic review literature also differs. It has been defined as less than 4 weeks', less than 6 weeks' and less than 12 weeks' duration. Subacute sciatica is usually between 6 and 12 weeks' duration. Chronic sciatica is greater than 12 weeks' duration. In this protocol, symptoms less than 4 weeks' duration are defined as acute.

The prevalence of lumbosacral radiculopathy has been estimated at $3 \%$ to $5 \%,{ }^{6}$ whereas referred leg pain is much higher. ${ }^{4}$ In an inception cohort of 1172 patients with acute low back pain presenting to primary care settings in Australia, $25 \%$ had leg pain. ${ }^{7}$ The majority of participants $(72 \%)$ with acute sciatica recover completely by 12 months. ${ }^{7}$ In another study, $50 \%$ of patients with acute sciatica recovered within 4 weeks. However, $30 \%$ had persistent leg pain and disability at 12 months. ${ }^{8}$

Patients with acute sciatica are treated with a combination of paracetamol, opiate analgesia, non-steroidal anti-inflammatory drugs (NSAIDs), ${ }^{9} 10$ pregabalin and physiotherapy, although a systematic review of pharmacological therapy that included NSAIDs, opioid analgesics, antidepressants, anticonvulsants, muscle relaxants and opioid analgesics showed no effect or only small effects in acute, subacute and chronic sciatica. ${ }^{11}$ Neuropathic symptom modifiers such as pregabalin have also recently been shown to be ineffective. ${ }^{12}$

During the 1970 s, failure of conservative management in sciatica and the desire to avoid surgery led to interventional procedures, including epidural steroid injections (ESIs). There are three approaches for ESIs: caudal, interlaminar and transforaminal. The transforaminal approach deposits steroid directly near the ventral epidural space at the affected unilateral nerve root level. Evidence for the superiority of the selective transforaminal approach versus the caudal and interlaminar is generally indirect ${ }^{13}$ as there are few high-quality head-to-head studies. ${ }^{14}$ Selective fluoroscopy (with or without CT-guided fluoroscopy) transforaminal epidural steroid injection (TESI) with local anaesthetic, colloquially described as a 'spinal perineural steroid injection', is increasingly being used in the management of patients with acute sciatica in hospital and community settings in the absence of any RCTs undertaken to evaluate the benefit of this procedure in patients with acute sciatica. There are no Cochrane reviews on the management of acute sciatica with epidural steroids of any route. ${ }^{15}$ In reviews of ESIs (caudal, laminar or transforaminal) in sciatica of any duration, not surprisingly, given the heterogeneity of patient populations, interventions, study design and study conduct, conclusions vary considerably. Two recent meta-analyses of epidural steroids in subacute and chronic sciatica ${ }^{1316}$ conclude that treatment effects are small and of only short duration.
The first transforaminal approach RCT was published in $2000 .{ }^{17}$ Five RCTs have been published ${ }^{18-22}$ that have had low risk of bias from random sequence generation and participant and personnel blinding. These RCTs show considerable heterogeneity in study design. All RCTs except one required a symptom duration of at least 4 weeks prior to recruitment. No RCT used CT fluoroscopy. All but one RCT required MRI evidence of disc herniation. ${ }^{17}$ Two studies excluded patients with evidence of foraminal stenosis. ${ }^{20} 22$ Three studies did not report neurological features. ${ }^{19} 2122$ All studies included an epidural control, but only one study also included a non-epidural control. ${ }^{20}$ Only two studies clearly specified the primary endpoint, ${ }^{20}{ }^{21}$ but these two studies had incomplete follow-up as they did not obtain further data on patients who failed to achieve a $50 \%$ reduction of pain 4 weeks after the last procedure. Where epidural saline was used as an epidural control, speculated mechanisms for a therapeutic effect include washout of inflammatory cytokines, lysis of inflammatory-mediated adhesions and enhanced blood flow to ischaemic nerves. ${ }^{20}$

Harms have been reported with TESIs ${ }^{23}$ including infection and bleeding. In 2014, the Food and Drug Administration issued a letter of warning that injection of corticosteroids into the epidural space of the spine may result in rare but serious adverse events, including 'loss of vision, stroke, paralysis, and death'. ${ }^{24}$ The risk is greater for particulate versus non-particulate steroids and in cervical versus lumbosacral epidurals. Recently, a consensus opinion paper was published on safeguards to prevent neurological complications after ESIs. ${ }^{25}$ The clinical considerations were based on conventional fluoroscopy with contrast and not with CT fluoroscopy. RCTs show no difference in efficacy between particulate and non-particulate steroids. ${ }^{26-28}$

Unlike epidural steroids, systemic steroids have been studied in acute as well as subacute sciatica. A meta-analysis of seven small of studies of variable quality of intramuscular (IM), intravenous and oral steroids found steroids were not superior to placebo and had more adverse events. ${ }^{29}$ Adverse events, however, were clearly related to the very high dose of dexamethasone used in three of the seven studies $(120 \mathrm{mg}$ of dexamethasone in 3 days, which is the equivalent of $800 \mathrm{mg}$ of oral prednisone). In another systematic review ${ }^{11}$ of three studies of acute sciatica using smaller doses of steroid, a significant effect on short-term overall pain and leg pain was found. A RCT of IM steroid versus IM saline failed to show a difference in leg pain scores. ${ }^{20} \mathrm{~A}$ blinded RCT reported that intravenous dexamethasone $(8 \mathrm{mg})$ improved pain scores at 24 hours and reduced emergency department (ED) length of stay compared with placebo. There was no difference at 6 weeks. ${ }^{30}$ No CT/MRI imaging evidence was required. A recent blinded RCT of patients of oral steroids (prednisone $60 \mathrm{mg} 5$ days, $40 \mathrm{mg} 5$ days and $20 \mathrm{mg} 5$ days) with sciatica less than 12 weeks' duration showed an improvement in function at 3 weeks and 52 weeks but no improvement in pain. ${ }^{31}$ 
In summary, there are two issues that are relevant that provide the rationale for this pilot/feasibility study: (1) the condition under study, that is, acute, subacute or chronic sciatica, and (2) the route of interventional procedure (caudal, interlaminar and fluoroscopic transforaminal epidural (the last with or without CT guidance)) or systemic route. There are no RCTs in acute sciatica published using steroid epidurals of any type. There are RCTs in acute sciatica with systemic steroids. In subacute and chronic sciatica, there are no RCTs that have used selective CT fluoroscopy transformational steroid injection, indicative of the fast pace of changing technological procedural interventions without RCT evidence. Arguably, steroids may be more effective for sciatica when provided in the acute setting, but this should be subjected to rigorous evaluation. In Australia, selective transforaminal epidural steroids is guided by CT fluoroscopy, therefore is performed by interventional radiologists. Given their use and perceived effectiveness, and the costs and potential harms associated with their use, there is an identified need to properly evaluate the use of epidural and systemic steroids in acute sciatica in adequately controlled trial designs with a control arm for the route of procedure. Furthermore, given that there is a rationale for the benefit of epidural saline in acute sciatica, epidural steroid could be directly compared with epidural saline to evaluate pharmacology versus a simple physical washout of inflammatory cytokines, lysis of inflammatory-mediated adhesions and enhanced blood flow to ischaemic nerves.

There is a clear advantage of directly comparing different interventions in a single RCT. These advantages include improving internal validity, marginally reducing sample size and limiting heterogeneity by standardising assessments and conduct procedures. However, there are also disadvantages such as longer time to trial recruitment, therefore longer time to trial completion, more exclusion criteria because of differing interventions and difficulty explaining design to participants.

\section{METHODS/ANALYSIS}

\section{Study objectives}

Primary objective

Our aim was to undertake a pilot/feasibility study of patients with acute sciatica designed to evaluate the feasibility of a blinded four-arm RCT of (1) selective CT fluoroscopy transforaminal epidural steroid (arm 1), (2) selective CT fluoroscopy transforaminal epidural saline (arm 2), (3) 15 days of a tapering dose of oral steroids (arm 3) and (4) a sham epidural and oral placebo control (arm 4). This feasibility study is designed to evaluate head-to-head, route versus pharmacology of corticosteroid intervention by comparing epidural steroid with systemic steroids, and epidural steroid with epidural saline and includes blinding with oral placebo and sham injection across all arms. The primary outcome measure is the Oswestry Disability Index (ODI) at 3 weeks. The primary analysis is comparison of CT fluoroscopy-guided transforaminal lumbar epidural steroid versus sham injection (arm 1 vs arm 4 in figure 1, study design).

The pilot/feasibility study will evaluate the following issues: rate of recruitment, study conduct including randomisation allocation concealment, preparation of interventions, choice of procedural corticosteroid and local anaesthetic, blinding, efficient organisation of initial assessments, diagnostic imaging and ensuring efficient study processes across public/private hospital inpatients, emergency department/room (ED/R) presentations and general practice visits, and timeliness of providing the intervention within the 4-week acute sciatica requirement. Rate of recruitment is important particularly where there already is an expectation of treatment benefit of 'spinal perineural steroid injections' by healthcare professionals and patients.

This pilot/feasibilitystudy is a single-centre Human Research Ethics Committee (HREC) study, but includes recruitment from multiple sources, and the interventions will be delivered in public hospital, private hospital and community radiology practices. The recruitment of participants and the delivery of the interventions have been designed to identify feasibility issues given these different settings.

\section{Secondary objectives}

1. Obtain preliminary results from this RCT, which will be used to calculate the sample size and power calculations for a full-scale study to determine which treatments currently used in the management of acute lumbosacral radiculopathy of less than 4 weeks' duration is the most effective in reducing pain and disability in the short term and prevent progression to persistent or recurrent lumbosacral radiculopathy in the long term.

2. Evaluate the adequacy of outcome measures in acute sciatica, where pain, sensory and motor neurological symptoms all cause distress and disability, and where pain caused by nerve root irritation often progresses to loss of pain and may be replaced by sensory loss or weakness from nerve root conduction impairment. The importance of describing this multifactorial pathology and how it impacts the primary endpoint, the ODI, has substantive importance regarding the optimal primary and secondary endpoint for use in a full-scale RCT. Other outcome measures will also be evaluated such as confounding by medication use and taper, protocol compliance and burden, confounding by modification of activities and need and timing of rescue procedures.

3. Although this is a feasibility study, for transparency the following are the prespecified hypotheses for powering a full-scale RCT. In patients with acute sciatica, selective CT fluoroscopy transforaminal lumbar epidural steroid (arm 1) is (a) superior to control (arm 4) and (b) non-inferior to a 15-day tapering dose of oral dexamethasone (arm 3) in reducing short-term pain and disability (after 3 weeks) as determined by the ODI. Further information regarding hypotheses and sample size is described in the sample size section. 
Recruitment of patients with acute sciatica ( $<21$ days)

Hospital emergency department, hospital in-patients, community GP and specialist referrals

Screen patient for eligibility

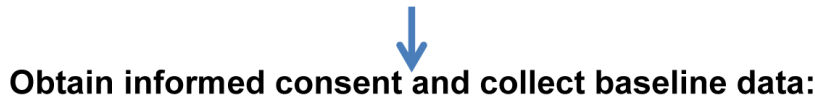

PROs", musculoskeletal \& neurological examination, CT/MRI imaging, safety blood tests

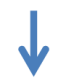

Intervention allocation in radiology suite

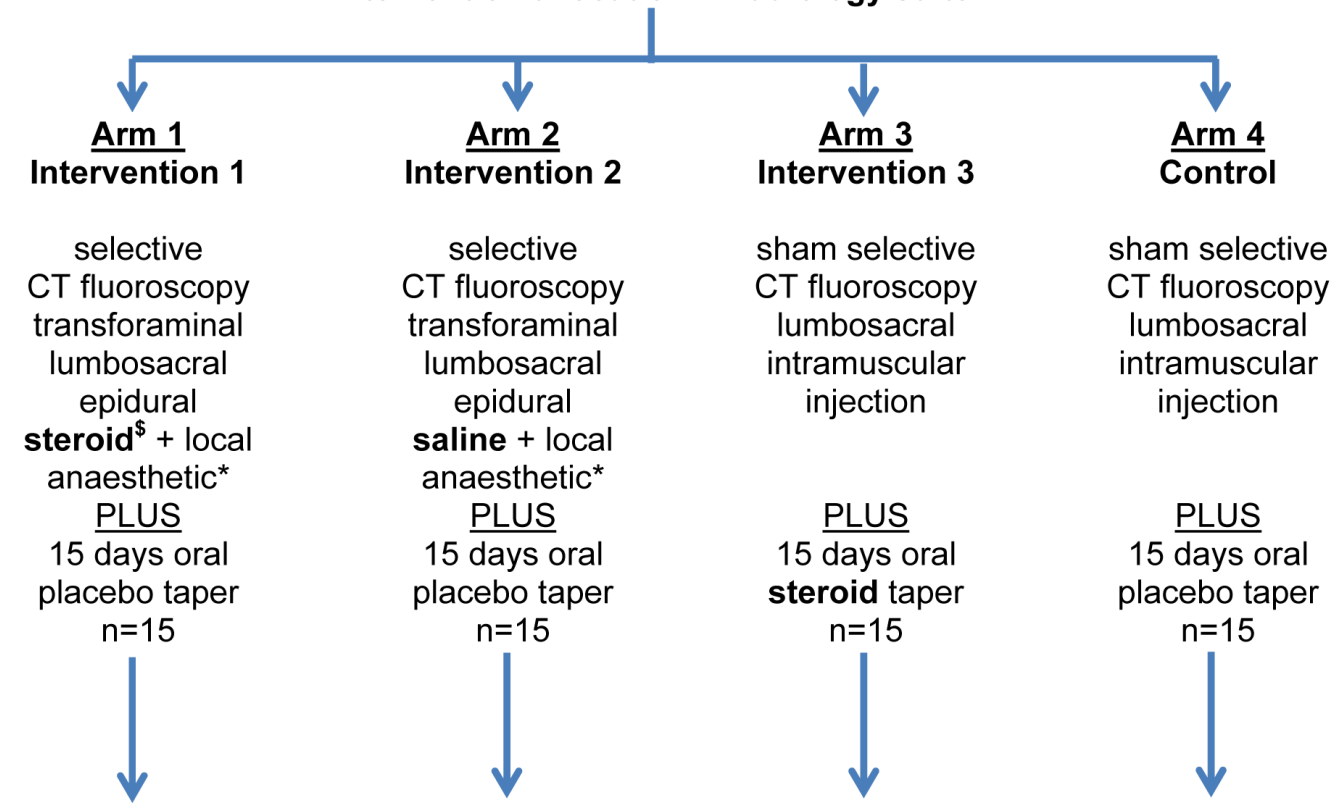

Follow-up for 48 weeks

Patient Questionnaires, musculoskeletal \& neurological history and examination Primary Endpoint: 21 days after procedure intervention using the Oswestry Disability Index

Figure 1 Study flow chart

\section{Participants, interventions and outcomes}

The study setting is the rheumatology service at a large teaching hospital in Sydney, Australia. The teaching hospital services a population of about 1 million of Southern Sydney. The eligibility criteria are as follows:

Inclusion criteria

i. Leg pain of any description with clinical findings consistent with single-level radiculopathy.

ii. Minimum symptom duration $>72$ hours.

iii. Maximum symptom duration $<3$ weeks to ensure symptom duration at randomisation is $<4$ weeks.

iv. No previous episode of same-level radicular pain in the previous 6 months.

v. Score at $>30$ on the ODI.

vi. Imaging (MRI and/or CT) indicating herniated disc or foraminal stenosis or both, concordant with the level indicated by history and physical examination.

vii. Age at least 18 years.
Exclusion criteria

i. Previous transforaminal epidural steroids at any level in the last 12 months.

ii. Previous oral steroids in the last 12 months.

iii. Any lumbar surgery at same level, or above or below the level at any time.

iv. Previous lumbar surgery at any other level to that in (iii) within the last 12 months.

v. Pregnancy or lactation/breast feeding.

vi. Direct indication for neurosurgery (eg, cauda equina syndrome or progressive motor loss, ie, $\leq 3 / 5$ power).

vii. Inability to read or understand English.

viii. Any serious medical or psychiatric condition that may interfere with participation or outcome assessment such as need for uninterrupted anticoagulation, spinal fracture, active infection or metastatic disease suspected, active cancer, poorly controlled diabetes or patients with diabetes on any insulin, uncontrolled 
hypertension (systolic blood pressure $>180$ or diastolic blood pressure $>110$ within 30 days of randomisation date), active peptic ulcer disease, history of intolerance to steroid therapy, previous or current psychiatric history of bipolar disease, or secondary gain such as anticipated or ongoing legal proceedings, and history of substance abuse.

ix. No other pathology likely to explain condition (eg, Guillain-Barre Syndrome, vasculitis).

Both MRI and CT scans are acceptable for entry criteria. If CT is equivocal regarding pathology or level, then the patient will proceed to MRI, or the patient is not included in the study. Scans are performed without contrast. All potential participants will be reviewed by a study physician (rheumatologist) who will undertake a history and physical general, musculoskeletal and neurological examination to ensure inclusion and exclusion criteria and exclude 'red flags' and alternate diagnoses. Full laboratory examination of safety includes full blood count, $\mathrm{C}$ reactive protein, erythrocyte sedimentation rate, coagulation profile, electrolytes, urea, creatinine, liver function tests and fasting blood glucose. Patients who can cease antiplatelet and anticoagulant medications safely will be given instructions on how to do so, or are excluded. The CT and/or MRI images are reported by an experienced radiologist who is unaware of the study, and the results are discussed with the participant and their treating physician. If the report is unclear, the images are reviewed by an independent radiologist at a radiology meeting to clarify imaging pathology. If imaging pathology remains unclear, then eligibility is not met. The images are also reviewed by the interventional radiologist prior to the procedure (see Implementation of interventions section). If the interventional radiologist cannot confirm the specified imaging pathology, the procedure is aborted and the principal investigator is contacted.

\section{Interventions}

The interventions are as follows and also summarised in table 1 and figure 1.

Table 1 Summary of the experimental interventions by arm

\section{Arm}

Arm 1

Intervention 1

Injectable dexamethasone and lignocaine OR betamethasone and bupivacaine selective CT fluoroscopy-guided transforaminal lumbar epidural steroid

\section{Experimental intervention}

Drug: betamethasone OR dexamethasone injectable

Procedural agents

The steroid and local anaesthetic preparation is determined by interventional radiologist's preferences regarding the use of particulate or non-particulate steroids

Dexamethasone $4 \mathrm{mg}(1 \mathrm{~mL})$ is a non-particulate corticosteroid and is used with the local anaesthetic lignocaine $1 \%(1 \mathrm{~mL})$

Betamethasone sodium phosphate/acetate $5.7 \mathrm{mg} / \mathrm{mL}$ injectable is a particulate corticosteroid and is used with the local anaesthetic bupivacaine $0.5 \%$ ( $1 \mathrm{~mL})$. Other name: celestone chondrase $5.7 \mathrm{mg}$ / $\mathrm{mL}$ injectable suspension

Other: oral placebo

The oral placebo is a gelatine capsule packed with filler

Arm 2

Intervention 2

Normal saline, $0.9 \%$ injectable solution with either bupivacaine or lignocaine selective CT fluoroscopyguided transforaminal lumbar epidural normal saline
Drug: normal saline, $0.9 \%$ injectable solution

Procedural agents

The local anaesthetic preparation used with the normal saline, 0.9\% injectable solution will be standardised to replicate current radiology interventional practices: either local anaesthetic bupivacaine $0.5 \%$

$(1 \mathrm{~mL})$ or local anaesthetic lignocaine $1 \%(1 \mathrm{~mL})$

Other: oral placebo

The oral placebo is a gelatine capsule packed with filler

\section{Arm 3}

Intervention 3

Dexamethasone oral capsule 15 day tapered dosing as follows: (1) days 1-5, $4 \mathrm{mg}$ morning and evening; (2) days 6-10, 2 mg morning and evening; and (3) days 11-15, $1 \mathrm{mg}$ morning and evening

\section{Drug: dexamethasone oral tablet}

Dexamethasone oral tablet: 15-day taper dosing is days $1-5,8 \mathrm{mg}$ (4 mg morning and evening); days 6-10, $4 \mathrm{mg}$ (2 mg morning and evening); and days 11-15, 2 mg (1 mg morning and evening). The dexamethasone is overencapsulated in a gelatine capsule that is identical to the placebo capsule in appearance
Other: sham injection

The sham injection procedure is needle placement down to muscle at the designated spinal level and no injection of any fluid.

\section{Arm 4}

\section{Control}

Sham injection and oral placebo: CT/fluoroscopy-guided (parameters set to zero) transforaminal lumbar sham (needle placement down to muscle and no injection of any fluid) AND placebo oral tablets taper
Sham injection and oral placebo

The sham injection procedure is needle placement down to muscle at the designated spinal level and no injection of any fluid. The oral placebo is a gelatine capsule packed with filler 


\section{Procedural interventions}

Once the specific spinal nerve pathology has been selected clinically and on imaging (eg, right S1 nerve root at L5/S1 intervertebral space), all participants are given an injection of local anaesthetic (lignocaine or bupivacaine) into the skin and subcutaneous tissue at this selected site.

Participants in arm 1 will receive selective CT fluoroscopy transforaminal epidural dexamethasone $4 \mathrm{mg}$ (1 mL), a non-particulate corticosteroid with the local anaesthetic lignocaine $1 \%(1 \mathrm{~mL})$. However, if participants are an inpatient at St George Hospital, they will receive betamethasone $(1 \mathrm{~mL})$ as celestone chondrose $5.7 \mathrm{mg} / \mathrm{mL}$, a particulate corticosteroid with the local anaesthetic bupivacaine $0.5 \%(1 \mathrm{~mL})$. This is at the direction of two interventional radiology investigators who have differing preferences regarding procedural agents. The interventional radiologist and their preference is known and will be addressed in the hierarchical linear model analysis.

Participants in arm 2 will receive selective CT fluoroscopy transforaminal epidural $0.9 \%$ normal saline $(1 \mathrm{~mL})$ and lignocaine $1 \%(1 \mathrm{~mL})$ unless they are hospital inpatients in which case they will receive bupivacaine $0.5 \%$ as the local anaesthetic agent. The saline epidural has two purposes in this pilot/feasibility study. There is no consensus in the literature regarding the optimal control for the evaluation of epidural steroids. ${ }^{32}$ Moreover, there is some evidence that it has a therapeutic effect. ${ }^{20}$ Therefore, this pilot/feasibility study is designed to explore these issues by including both epidural saline arm (arm 2) and a sham injection (arms 3 and 4).

Participants in arms 3 and 4 will receive sham-selective CT fluoroscopy intramuscular injection with needle placement down to muscle layer and no injection of any fluid. The intervention is performed by an experienced interventional radiologist. The intervention radiologist is not blind to the procedure (see Masking/blinding section for more information).

\section{Oral intervention}

The oral steroid is dexamethasone. The 15-day taper dosing is (1) $4 \mathrm{mg}$ at 08:00 and 18:00 days $1-5$, (2) $2 \mathrm{mg}$

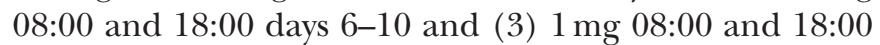
days 11-15. Dexamethasone has a longer biological halflife than prednisolone. The oral interventions are overencapsulated in gelatine capsules packed with sucrose and lactose. The placebo is sucrose and lactose only. Participants in arm 3 receive the oral dexamethasone capsules, and participants in arms 1, 2 and 4 receive the placebo capsules. Dexamethasone and placebo capsules have identical appearance and are prepared by a compounding pharmacist. The capsules are placed in three plastic bottles with clearly labelled instructions. At each telephone or in-person contact, treatment adherence is monitored.

\section{Concomitant management and interventions}

All participants have concomitant usual care therapy as directed by the treating physician(s) with analgesics, NSAIDS, pregabalin and physical therapies. All concomitant therapy will be recorded at each visit. Rescue therapy includes CT fluoroscopy transforaminal epidural of steroid and neurosurgery.

\section{Outcomes}

A recent publication on core outcomes domains for clinical trials in non-specific low back pain recommended physical functioning, pain intensity and health-related quality of life. ${ }^{33}$

\section{Primary outcome measure}

The Oswestry Disability Index (ODI) version $2.0^{34}$ is the primary outcome measure. The ODI is a functional status measure specifically developed for disorders of the spine and has been used in most RCTs of sciatica ${ }^{35}$ (see table 2). It is a 10-domain 2-page 5 min questionnaire with ordered 6-response-item (0-5) scales for each question. The questions address domains of pain, physical functioning, sleeping, home/work functioning and impact on social life. The scores are summed, then doubled and the final score is $0-100$. The ODI will be administered at eligibility, baseline/randomisation (day 0 ), days 1-7, weeks 2, 3, 6, 12, 24 and 48. This will be administered at visits, phone or mail. The primary analysis is the short-term outcome, reduction of disability at 3 weeks on the ODI. The secondary analysis is the longterm outcome, reduction of disability at 48 weeks on the ODI.

\section{Secondary outcomes}

Numerical Rating Scale (NRS) for leg pain is the main secondary outcome. A measure of leg pain is included in all studies of sciatica. The NRS is a validated ${ }^{36} 11$-point scale. Participants will be asked to rate their average leg pain over the preceding 24 hours. Zero represents 'no leg pain' and 10 represents 'worst imaginable pain'. Although the Visual Analogue Scale (VAS) is a more frequently included measure, unlike the VAS, the NRS can be verbally administered by phone. This will be administered at eligibility, baseline/randomisation (day 0), days 1-7, weeks 2, 3, 6, 12, 24 and 48.

Numerical Rating Scale (NRS) for back pain. The severity of back pain may differ to that of leg pain, so both measures are needed. It is rated as an average over the preceding 24hours and will be administered at eligibility, baseline/ randomisation (day 0), days 1-7, weeks 2, 3, 6, 12, 24 and 48.

Pain DETECT Questionnaire. ${ }^{37}$ At eligibility, baseline/ randomisation (day 0 ), days $1-7$, weeks $2,3,6,12,24$ and 48.

Short Form 36 (SF-36) questionnaire ${ }^{38}$ evaluates health-related quality of life and will be administered at eligibility, baseline/randomisation (day 0 ), day 1 , day 7 , weeks 3,6 , 12,24 and 48 . 
Table 2 Schedule of enrolment, interventions and assessments

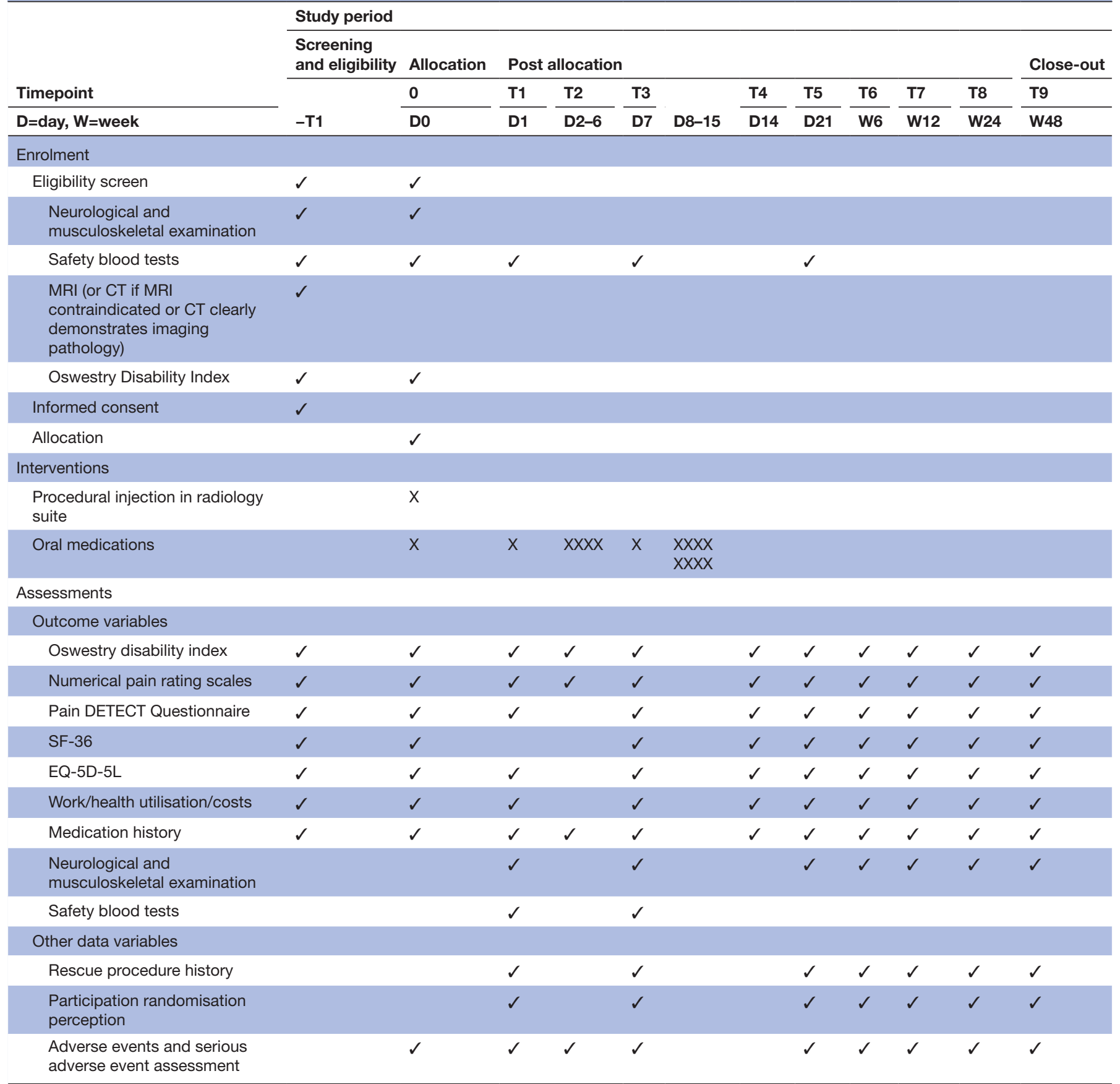

SF-36, Short Form 36.

Lumbosacral and lower limb musculoskeletal and neurological history and clinical examination at eligibility, baseline/ randomisation (day 0), day 1, day 7, weeks 3, 6, 12, 24 and 48. This includes inspection of gait, lumbosacral spine and lower limbs for scoliosis, asymmetry, loss of lumbar lordosis, abnormal gait and stance, weakness, muscle wasting, muscle fasciculation, palpation of lumbosacral spine for tenderness and rigidity, movement of lumbosacral spine in flexion and extension, hip, knee and ankle range of movement, straight leg raise and femoral stretch test. Neurological examination of lower limb includes further inspection, examination for tone (normal, increased, decreased), clonus (present, absent and beats of clonus if present), power $(0,1,2,3,4,4+$ and 5 out of 5 ) for 12 lower limb movements (hip abduction, adduction, flexion, extension, knee flexion and extension, ankle dorsiflexion, plantar flexion, inversion and eversion, big toe extension and flexion), knee and ankle reflexes (increased, normal, decreased, absent), plantar reflexes (normal, up-going, equivocal, no response), and pinprick, light touch, proprioception and vibration sensory examination.

Work and health utilisation measures at eligibility, baseline/randomisation (day 0 ), day 1 , day 7 , weeks 3,6 , 
12, 24 and 48. These will include days missed from paid employment (if applicable) because of sciatica, use of health services such as doctor, other healthcare provider-related visits (eg, acupuncture, chiropractic), injection procedures and neurosurgery. This information will be obtained by interview at each visit and is documented in the case report form developed for the study.

Demographic and socioeconomic measures measured at baseline include age, gender and occupation/previous occupation.

Imaging findings on CT and/or MRI will be used to define the site, level, type and degree of pathology using classification systems for disc herniation ${ }^{39}$ and severity of nerve root compression. ${ }^{40}$ These data will be used to determine imaging predictors of response.

\section{Medications}

Use of all other medications including analgesics, NSAIDs, opiates, gabapentin and pregabalin will be documented at every visit.

\section{Economic evaluation}

Outcomes for an economic evaluation will also be collected in this feasibility study. The feasibility of a cost-effectiveness analysis will be undertaken using the ODI and a cost-utility analysis ${ }^{41}$ using the EQ-5D-5L for incremental costs per quality-adjusted life year (QALY). ${ }^{42}$ The EQ-5D-5L questionnaire will be administered at eligibility, baseline/randomisation (day 0 ), day 1 , day 7, weeks 3, 6, 12, 24 and 48. Work and health utilisation measures described above will also be collected. Costs within each randomised arm will be assessed in terms of hospital, healthcare visits, investigations, such as CT and MRI imaging, procedure costs and medications costs. These direct costs are determined with Diagnosis Related Groups cost weights for hospital inpatients, and for outpatients by the Australian Medical Benefits Scheme standard fees, and the Australian Pharmaceutical Benefits Scheme. These costs are determined by the Australian Pharmaceutical Benefits Advisory Committee (PBAC) Manual of Resources items and their associated costs used for economic analyses. ${ }^{43} 44$ The PBAC does not require questionnaires of productivity ${ }^{434}$ such as the PRODISQ ${ }^{45}$ and similar questionnaires of resource utilisation. ${ }^{46}$

Adverse events will be collected at day 1 , day 7 , weeks 3, 6, 12, 24 and 48. These will include steroid adverse effects (blood pressure, blood glucose, changes in mood and sleep) and procedural adverse effects (headaches, bleeding) and information about additional procedures, surgery and hospitalisations.

\section{Sample size}

Most trials of subacute and chronic sciatica of a selective CT fluoroscopy TESI have a sample size of 30 participants per arm. The primary outcome in this pilot/feasibility study is the ODI at 3 weeks comparing epidural steroid and sham injection (arm 1 vs arm 4). With 15 participants per arm, there is $85 \%$ power to detect a difference of 17
ODI points between these two arms, given a SD of change of ODI of 15.1 points. ${ }^{31}$ Statistical test on which calculation is based is the independent two-sample t-test with a two-tailed alpha of 0.05 (Stata V.14). This is a total of 60 participants in this pilot/feasibility study. This is sufficient to evaluate feasibility of the study design, study conduct and determine sample size for a full-scale multicentre study. However, this ODI difference is a large unrealistic effect. The minimum clinically important difference in ODI scores in one study was 7.0 points, ${ }^{47}$ and an international consensus group found empirical evidence of 4 to 15 ODI points ${ }^{48}$ and recommended a cut-off value of 10 ODI points. In a full-scale study recruiting participants with acute sciatica of less than 4 weeks' duration, an ODI difference of at least 10 ODI points is very reasonable. A sample size of 49 participants per arm would provide $90 \%$ power to detect a minimum clinically important difference of 10 ODI points assuming a SD of 15.1 with a two-tailed alpha of 0.05 (Stata V.14). Allowing for $20 \%$ dropout (which at 3 weeks is unlikely but at 48 weeks is more likely), 236 participants would be recruited, 59 to each arm. Although there are six possible comparisons in a four-arm trial, controlling for type 1 error rate is not needed when several different experimental arms are compared with the control. ${ }^{49}{ }^{50}$ Therefore, no multiplicity adjustment is needed for (1) comparison I-arm 1 versus arm 4 (epidural steroid is superior to control), (2) comparison II-arm 2 versus arm 4 (epidural saline is superior to control) and (3) comparison III-arm 3 versus arm 4 (oral steroid is superior to control). However, in order to proceed to comparison IV, arm 1 versus arm 3 (epidural steroid is superior to oral steroids), we must first demonstrate that comparisons I and III were statistically significant, and there must be a type 1 error consideration. ${ }^{51}$ Furthermore, if the hypothesis is that oral steroid is non-inferior to epidural steroids, then the ignorable difference must also be prespecified. The pilot/feasibility study will provide data that will be helpful in determining these sample size calculations. The feasibility study will be informative regarding the estimated mean difference in this population, its SD and pattern of missing data at each of the study visits.

\section{Recruitment processes}

Participants will be recruited from (1) EDs of public hospitals, (2) current inpatients of public and private hospitals and (3) referral from community general practitioner (GP) or medical specialist (rheumatologist, neurosurgeon or orthopaedic surgeon) from the Sydney metropolitan area around St George Hospital. It is anticipated that the majority of participants will be recruited from ED presentations and GPs. Participants with sciatica symptoms less than 21 days' duration are screened so that participants can be evaluated and undergo the allocated intervention within the 4-week eligibility criteria.

St George Hospital ED as well as GPs and relevant specialists in the neighbouring geographical area (population 270000 ) serviced by this hospital area have been 
provided information about the SCIATICA study, the inclusion/exclusion criteria, explanation of the trial rationale and the opening of a daily acute sciatica clinic at St George Hospital centre as the portal of entry for trial patients.

Participants presenting to the ED with acute sciatica are assessed according to ED's usual procedures and staff admit or discharge patients according to their usual care pathway. If the ED does not admit a potential acute sciatica participant, a study clinician is contacted by phone Monday-Friday 09:00 to 17:00 (business hours) and a referral is faxed. Out of business hours, a referral is faxed to the acute sciatica clinic, which is processed the next business day (see below). All referred participants are given a brochure by the referring ED clinician outlining the study. The acute sciatica clinic is also available for urgent referrals from community GPs and specialists. This is by fax or by telephone. These referred participants are also given a brochure by their referring clinician. All referred potential participants are logged.

Within 1 to 3 days, Monday to Friday, all referred participants are contacted by telephone by a study clinician and a telephone history is obtained to ascertain suitability regarding inclusion and exclusion criteria. Where eligibility is clear or indeterminate, an eligibility visit is organised within the next couple of days. At this visit, a full history and examination, musculoskeletal and neurological, is conducted to determine underlying pathology, and if acute sciatica is likely, then lumbosacral imaging preferably with MRI imaging and blood pathology is requested. Patients complete routine clinical practice questionnaires as part of clinic audit including ODI, SF-36 and EQ-5D-5L. Conservative therapy is initiated (medication/physiotherapy) as appropriate. Potential participants are provided with the participant information and consent form and further information regarding the RCT if eligibility criteria are likely. Once imaging and pathology becomes available, the participant is contacted and informed of the results. If $\mathrm{s} /$ he meets the criteria, $\mathrm{s} /$ he is invited to participate in the RCT. At one of the visits prior to randomisation, all participants are reviewed by the principal investigator to ensure that all eligibility criteria are met. This includes a full general, musculoskeletal and neurological history and clinical examination and confirmation of imaging. If eligibility criteria are met and the participant agrees to participate, then the participant proceeds down study pathway. Processes are in place to ensure that enrollees, if they agree to participate, are safely fast-tracked to randomisation and RCT interventions.

If patients do not agree to participate in the RCT, they can either decide to continue their management in the acute sciatica clinic, and if their GP is willing, then the patient's ongoing management is determined by the rheumatologists who run the acute sciatica clinic. If the patient wishes to be managed by their GP, a letter from the acute sciatica clinic is sent to the GP to facilitate management. The patient has the option of returning to the acute sciatica clinic for further management or advice as needed. A log of potential participants who decline or are ineligible for any reason is kept for later evaluation consistent with Consolidated Standards of Reporting Trials (CONSORT) guidelines. ${ }^{52}$ Reason for rejection or refusal will be recorded if available as well as age, gender, race/ethnicity and ODI score. If the participant does not wish to participate in the RCT but wish to be managed in the acute sciatica clinic, they are included in a clinical audit of the management of acute sciatica. The management is determined in consultation with the patient and is generally conservative therapy unless there is severe pain and progressive functional disability preventing return to work or normal activities, progressive motor weakness or features on the MRI imaging that suggest that neurosurgical review is needed.

The participant may clearly not meet the eligibility criteria at telephone screening. If patient safety is not an urgent consideration, patients who have anticipated or ongoing legal proceedings, need uninterrupted anticoagulation or active cancer (as exclusion criteria) are not progressed to the eligibility visit but are asked to see or return to their treating doctor. Participants who do not have any leg pain are also asked to see or return to their treating doctor. However, if a referred patient has a history that suggests cauda equina syndrome or symptoms suggestive of malignant or infection-related pathology, the patient is seen urgently in the acute sciatica clinic and appropriate investigations and management are instituted.

If the potential participant is admitted to hospital with acute sciatica, the admitting team will contact the study investigators. Most patients with acute sciatica in our setting are either admitted under the general medical team, the rheumatology team or the neurosurgical team. The same processes are followed for in patients as described above for out patient referrals. Only a study investigator can consent a participant to participate in SCIATICA.

If the in-patient participant does not wish to participate, they are included in a clinical audit of the management of acute sciatica during the admission and the participant is continued to be managed according to the treating clinician. This is generally conservative therapy unless there is progressive severe pain and functional disability preventing discharge, progressive motor weakness or features on the MRI imaging that suggest that neurosurgical review is needed.

All participants are told that participation is voluntary and they can discuss participation with family, friends or their healthcare practitioners, and if they decide not to participate, it will not affect the treatment they receive now or in the future. They can have family and friends with them during the informed consent process. They can also withdraw from the study once it has started, at any time without having to give a reason. 


\section{Assignment of interventions}

Sequentially numbered, opaque and sealed envelopes contain the randomised intervention. Participants are randomly allocated $1: 1: 1: 1$ by computer-generated random numbers using permuted blocks stratified by duration of sciatica ( $\leq 2$ weeks, $>2$ weeks). The randomisation schedule including details of blocking schedule are held off-site by the randomised allocation sequence study investigator who is not involved in participant recruitment, assignment of interventions or data collection to ensure allocation concealment. This study investigator places the study medications and procedure instructions for each arm in separate opaque sealed envelopes. These two envelopes in turn are placed into a single larger opaque sealed envelope labelled with a sequential number and the randomisation number. The sealed envelopes are held in a locked cabinet until retrieved by the blinded study investigators who are involved in participant recruitment, provision of the study interventions, participant management and data collection. The acute sciatica clinic study investigators are blind to the study intervention.

\section{Implementation of interventions}

The day of study intervention implementation, the participant has safety bloods performed, unless eligibility safety bloods had occurred in the previous week. The participant completes the study questionnaires and the study clinician once more ascertains eligibility criteria by history and examination immediately in the morning before attending the radiology suite. If the criteria are still met, the study clinician indicates the exact site of the CT fluoroscopy transforaminal epidural on a request form that is provided to the interventional radiologist. For example, "perform a selective CT fluoroscopy transforaminal epidural of corticosteroid and local anaesthetic at L5/S1 targeting the right S1 nerve root'. The MRI images are also provided to the interventional radiologist. The research officer retrieves the next in sequence numbered large opaque labelled sealed envelope. The research officer accompanies the participant, taking the interventional request, images (films or on CD) and large opaque labelled sealed envelope to the radiology suite. At the radiology suite, the research officer opens the sealed opaque envelope, gives the 'procedure' envelope with instructions to the radiologist and exits. The radiologist evaluates the MRI images, then opens the procedure envelope. It contains one of three instructions: (1) selective CT fluoroscopy transforaminal epidural steroid and local anaesthetic injection, (2) selective CT fluoroscopy transforaminal epidural normal saline and local anaesthetic injection or (3) intramuscular sham injection down to muscle layer but no injection of any fluid. The side (right or left) and lumbosacral level (eg, L5/S1) is determined by the radiology request form. The participant is positioned prone as per a CT fluoroscopy transforaminal epidural, the CT fluoroscope is positioned as if a CT fluoroscopy transforaminal epidural is performed, and local anaesthetic is injected into the skin and subcutaneous tissue. Radiologist and his staff maintain patient blinding. CT/ fluoroscopy-guided transforaminal lumbar epidural radiation parameters are set to reduce radiation dose. There is no radiation dose for CT/fluoroscopy-guided transforaminal lumbar sham injection because the parameters are set to zero although the machine is on. All CT fluoroscopy images are saved for further analysis.

At the end of the procedure, once outside the CT fluoroscopy room, the research officer gives the opaque envelope marked 'Dexamethasone or placebo capsules' to the participant and explains how the medications are to be taken over the next 15 days. There are three plastic bottles labelled days $1-5$, days $6-10$ and days $11-15$. The participant opens the day 1 labelled bottle and swallows the capsule. The participant continues to lie flat for at least 1 hour after the procedure, the participant is forbidden to drive for 24 hours and a person accompanies them home. The interventional radiology procedure report states that the participant had a procedure as part of the SCIATICA RCT, and to contact the chief investigator if there is a concern, a phone number is provided.

\section{Masking/blinding}

All personnel except the radiologist delivering the procedure and the investigator responsible for randomisation and preparing the interventions will be blind to the randomisation arm. The trial participant, study clinicians, research officers, participant's treating care providers, outcome assessors and data analysts are blind to the intervention assignments. In the event of a serious medical emergency during which the treating doctor must know in which arm the participant was randomised, the randomised code can be broken. Each participant is given a 24-hour emergency contact number and the principal investigator contacts the investigator who holds the randomisation schedule to determine the participant's allocated intervention.

\section{Data collection, management and analysis \\ Data collection methods}

Data on quality of outcome, baseline and other trial data are safeguarded with standardisation, assessor training and duplication of measurements and assessments by research officers administering the questionnaires and study clinicians undertaking the history and clinical examinations. All assessments are reviewed and the history and clinical findings confirmed by the principal investigator prior to final eligibility determination. Study clinicians meet every 2 weeks to discuss ongoing assessments, issues of standardisation, equivocal or unclear findings and/or any other concerns. All questionnaire data are scanned, with range checks for data values, and verified. Free-text data are scanned and verified. Clinical data are coded and verified. Participants' retention and complete follow-up is encouraged through contact by phone or text and visits are organised so that they are maximally convenient for 
participants. This often requires visits to be conducted at the end of the normal working day.

\section{Data/statistical analysis plan}

Although this is a pilot/feasibility study to evaluate several important clinical and trial design considerations, the following data analysis plan is proposed for transparency. In this feasibility study, treatment is analysed by intention to treat and the data analyst will be blind to arm allocation. A two-tailed $p$ value $<0.05$ is considered statistically significant. The primary analysis is an analysis of variance evaluating the effects of treatment on the ODI at week 3 , using treatment arm, baseline ODI and duration of symptoms in days as covariates. The primary comparison is epidural steroid versus control. However, similar analyses will be applied to the other treatment comparisons with control (epidural saline vs control, oral steroid vs control) without a type 1 error penalty. However, the epidural steroid versus oral steroid comparison will require type 1 error consideration. ${ }^{51}$ All comparisons are made at day 21 , where day 0 is the day of the procedural intervention immediately followed by the first dose of the oral intervention. Day 21 is the 3-week endpoint. Similar analyses will also be applied at the 6-week and 48-week endpoints for the ODI.

Multilevel linear mixed model will examine time trend by treatment arm interaction. This linear mixed model will be used to model ODI trajectory across all 10 timepoints by treatment arm, where treatment arm is a property of the persons and visit is nested within person. The random-effect portion of the model is time, which here is each measurement, treated in the model as monthly time intervals. Analyses will be undertaken unadjusted and adjusted for (1) medication use, (2) presence of a definite motor radiculopathy, (3) days from onset of sciatica pain to delivery of the intervention, (4) whether the imaging demonstrates a prolapsed disc, a sequestered disc or a extruded disc fragment, (5) whether imaging demonstrates bony/osteophytic narrowing of the neural exit foramen and (6) age. Missing data will be handled with multiple imputation, using iterative Markov chain Monte Carlo, which requires the assumption that the data are missing at random..$^{53}$ An intention-to-treat analysis with multiple imputation is the primary analysis; however, a completer's analysis will also be undertaken as a secondary analysis. The value of undertaking a feasibility study is that patterns and reasons of missing data that are not at random may be identified and in the fullscale study targeted efforts made to reduce this potential bias. There is no interim analysis.

Other outcome measures (NRSs, SF-36, EQ-5D and clinical data measured on a continuous scale) will also be analysed with multilevel mixed-effects linear regression. All analyses will be undertaken unadjusted and adjusted for other medication use, type of procedural steroid, presence of neurological signs and MRI findings with multivariate methods. A full description of neurological signs will be reported in tabular form and descriptive statistics.
Safety data will be analysed and reported in tabular form and with descriptive statistics.

\section{Economic evaluation}

This feasibility study will provide data to identify issues conducting an economic evaluation for the full-scale study. The rationale for undertaking an economic evaluation is to evaluate the feasibility of undertaking a prespecified cost-effectiveness economic evaluation in the full-scale study. In Australia, all drugs, and more recently, certain procedures, undergo a cost-effectiveness analysis to determine whether they will be subsidised by the Australian government. This is usually performed from the perspective of the healthcare sector rather than from the societal perspective. ${ }^{43}$ We will be following these guidelines. In this pilot/feasibility study, we will ascertain the feasibility of obtaining the outcome (including QALYs) and cost data in a valid manner, determine how much outcome and cost data are missing, and obtain estimates of mean and SD of outcomes and costs. The Consolidated Health Economic Evaluation Reporting Standards (CHEERS) ${ }^{41}$ statement checklist will also be followed to report the economic evaluation component in the full study.

In this pilot/feasibility study, all participants in all study arms have concomitant usual care therapy as directed by the treating physician(s) with analgesics, NSAIDS, pregabalin and physical therapies. Arm 4, the control arm, therefore is the usual care arm. In this pilot/feasibility study, the perspective of the health sector is undertaken using intention to treat. The incremental cost per point on the ODI or QALY (based on EQ-5D-5L) will be estimated as the ratio of the difference in average cost and ODI or QALY between intervention arms for three comparisons: (1) epidural steroid versus control, (2) oral steroid versus control and (3) epidural steroid versus oral steroid. Missing data will be imputed with iterative Markov chain Monte Carlo methods. Sensitivity analyses will be performed by converting the SF-36 to SF-6D QALYs to compare QALYs, as well as other sensitivity analyses as recommended by CHEERS.

\section{Patient and public involvement}

Patients and or public were not formally involved in the development of the research question and outcome measures. Patients were not involved in the design of this feasibility study. Patients were not involved in the recruitment to and conduct of this feasibility study. At the end of the study, a report of the study results will be provided to all study participants. In this feasibility study of a RCT, the burden of the intervention was not assessed by patients or the public.

However, the South Eastern Sydney Local Health District Human Research Ethics Committee (HREC/15/331/ POHW/586), which includes members of the public, assisted with the design and content of the patient information and consent form that was developed for this study. As a result of the committee's contribution, the revised patient information and consent form clearly 
provides the reason for undertaking the study, the outcome measures involved, explains the nature of the interventions and their burden, and clearly summarises overall study conduct.

\section{ETHICS AND DISSEMINATION \\ Ethics}

The study has been approved by South Eastern Sydney Local Health District Human Research Ethics Committee and is guided by a Data Safety and Monitoring Board and South Eastern Sydney Local Health District Human Research Ethics Executive (HREC15/331) Protocol version 3, 6 April 2016. Any changes to the protocol are reported to this committee.

\section{Data monitoring}

A data safety and monitoring committee (DSMC) will meet after the first 10 participants have been randomised to evaluate study conduct and safety. The DSMC will consist of the principal investigator (non-voting), an interventional radiologist, neurosurgeon, rheumatologist and general physician. Adverse event monitoring and withdrawal of participants are discussed. The DSMC will meet every 4 months. The DSMC will be provided blinded data, but unblinded data can be provided for a specific participant if requested by the committee. If requested, it will be provided by an investigator who holds the randomisation schedule.

\section{Harms}

CT fluoroscopy-guided transforaminal lumbar epidural steroid $(1 \mathrm{~mL})$ and local anaesthetic $(1 \mathrm{~mL})$ is used in the management of sciatica of all durations. The risks associated with this procedure include:

\section{Dural puncture}

The needle penetrates into the sac encasing the nerves within the spinal canal, causing leakage of fluid contained within the sac, known as cerebrospinal fluid. The risk of this procedure is approximately $1 \%$ and is treated with flat bed rest for 4 hours.

\section{Infection}

Most of these are minor (1\%-2\%), however can be serious $(<0.1 \%)$ requiring hospital admission, intravenous antibiotics and surgery.

\section{Bleeding}

This is rare although more common in patients with bleeding disorders and on 'blood thinning' medication. Patients who cannot cease their medications will be excluded from the study (eg, patients with mechanical heart valve, recent deep venous thrombosis and pulmonary embolus, recent cardiac stent). Otherwise, patients on warfarin have an International Normalised Ratio (INR) and depending on the value will be asked to cease the warfarin 5 days prior to the procedure and an INR will be checked the day before the procedure and the value must be $<1.5$. Pradaxa (dabigatran) must be ceased 3 days prior to the procedure, aspirin and platelet inhibitors (plavix, iscover, ticlopidine, persantin) ceased 7 days prior to the procedure, and clexane ceased 24 hours prior to the procedure. NSAIDs and COX2 inhibitors do not need to be ceased.

\section{Nerve damage}

From direct needle trauma, or as a consequence of the aforementioned complications, is rare.

\section{Stroke and spinal cord injury}

Most of the reported serious complications result from inadvertently injecting steroids with particulate matter into blood vessels close to the injection site, which can lead to brain or spinal cord injury. The risk of stroke or spinal cord damage from a TESI in the back is quite low when done under CT fluoroscopy.

The risks of high-dose short-term oral corticosteroids are more common (10\%-20\%) and include insomnia, nervousness, increased appetite, indigestion and headache. There are risks in patients with active peptic ulcer disease of perforation, worsening hypertension in patients with severe hypertension and hyperglycaemia in patients with poorly controlled diabetes or on insulin treatment. These patients are excluded from the trial. Patients who are on diet or oral hypoglycaemic medications will be monitored with blood tests to minimise risk of significant hyperglycaemia. However, these symptoms and abnormal blood tests will cease with stopping of treatment. There is no risk of suddenly stopping dexamethasone in this study as it is only being administered for 2 weeks.

It is important that women participating in this study are not pregnant or lactating as the study CT scan fluoroscopy radiation, although small, is not zero, and dexamethasone is secreted in breast milk.

An adverse event is any untoward medical occurrence in a participant that does not necessarily have a causal relationship with the study treatment. An adverse event can therefore be any unfavourable or unintended sign, symptom or condition and/or an observation that may or may not be related to the study treatment. A serious adverse event is any untoward medical occurrence that results in the following: death, is life-threatening, requires inpatient hospitalisation or prolongation of existing hospitalisation, persistent or significant disability/ incapacity or congenital/birth defect, and condition requiring unnecessary medical or surgical intervention. Solicited reporting of adverse events occurs days 1 to 7 , weeks 3, 6, 12, 24 and 48. Participants can also contact study investigators at any time if they have any concerns. All adverse events are reported to the principal investigator and all serious adverse events are reported to the DMSC and Human Research Ethics Committee.

\section{Auditing}

A study meeting to audit trial conduct occurs fortnightly. There is no independent trial audit other than that 
provided by the DSMC and that required by the Human Research Ethics Committee.

\section{Access to data and dissemination}

The investigators have access to the final trial dataset. There are no contractual agreements limiting access. Study results of this trial will be submitted for publication in a peer-reviewed journal. Individual-level data will be made available after the findings of the study have been published. These data can be used for individual patient data (IPD) meta-analyses or for further exploratory research. To obtain these data, please contact MNL.

Acknowledgements We would like to thank Dr Derek Glenn, Head, Department of Radiology, St George Hospital, Kogarah for assisting with the information regarding radiation safety; Dr Carl Bryant, Bryant Radiology, St George Private Hospital for undertaking the interventional procedures; Ms Sue Baker for developing the case report forms, setting up the database and assisting with the ethics application; and Ms Jenny Gu for editing the case report forms.

Contributors MNL conceived and designed the study. MNL and KRJ wrote the first draft of the protocol. PS, GP and JT critically reviewed the protocol for important intellectual content and approved the final version.

Funding This work was supported by The St George and Sutherland Medical Research Foundation (SSMRF), Development Grant Number 2016/13 (http://www. stgeorgemrf.com.au/2015/11/02/our-2016-grants/).

Disclaimer The sponsor had no role in the study design of this protocol and will have no role in the collection, management, analysis and interpretation of data; writing of the report; and the decision to submit the report for publication or authority over any of these activities.

Competing interests None declared.

Patient consent Obtained.

Ethics approval South Eastern Sydney Local Health District Human Research Ethics Committee HREC15/331.

Provenance and peer review Not commissioned; externally peer reviewed.

Open access This is an open access article distributed in accordance with the Creative Commons Attribution Non Commercial (CC BY-NC 4.0) license, which permits others to distribute, remix, adapt, build upon this work non-commercially, and license their derivative works on different terms, provided the original work is properly cited and the use is non-commercial. See: http://creativecommons.org/ licenses/by-nc/4.0/

(c) Article author(s) (or their employer(s) unless otherwise stated in the text of the article) 2018. All rights reserved. No commercial use is permitted unless otherwise expressly granted.

\section{REFERENCES}

1. Koes BW, van Tulder MW, Peul WC. Diagnosis and treatment of sciatica. BMJ 2007;334:1313-7.

2. Bogduk N. On the definitions and physiology of back pain, referred pain, and radicular pain. Pain 2009;147(1-3):17-19.

3. Stafford MA, Peng P, Hill DA. Sciatica: a review of history, epidemiology, pathogenesis, and the role of epidural steroid injection in management. Br J Anaesth 2007;99:461-73.

4. Valat JP, Genevay S, Marty M, et al. Sciatica. Best Pract Res Clin Rheumatol 2010;24:241-52.

5. Lin CW, Verwoerd AJ, Maher CG, et al. How is radiating leg pain defined in randomized controlled trials of conservative treatments in primary care? A systematic review. Eur J Pain 2014;18:455-64.

6. Tarulli AW, Raynor EM. Lumbosacral radiculopathy. Neurol Clin 2007;25:387-405.

7. Henschke N, Maher CG, Refshauge KM, et al. Characteristics of patients with acute low back pain presenting to primary care in Australia. Clin J Pain 2009;25:5-11.

8. Weber H, Holme I, Amlie E. The natural course of acute sciatica with nerve root symptoms in a double-blind placebo-controlled trial evaluating the effect of piroxicam. Spine 1993;18:1433-8.
9. Rasmussen-Barr E, Held U, Grooten WJ, et al. Nonsteroidal antiinflammatory drugs for sciatica: an updated Cochrane review. Spine 2017;42:586-94.

10. Rasmussen-Barr E, Held U, Grooten WJ, et al. Non-steroidal anti-inflammatory drugs for sciatica. Cochrane Database Syst Rev 2016;10:CD012382.

11. Pinto RZ, Maher CG, Ferreira ML, et al. Drugs for relief of pain in patients with sciatica: systematic review and meta-analysis. BMJ 2012;344:e497.

12. Mathieson S, Maher CG, McLachlan AJ, et al. Trial of pregabalin for acute and chronic sciatica. N Engl J Med 2017;376:1111-20.

13. Pinto RZ, Maher CG, Ferreira ML, et al. Epidural corticosteroid injections in the management of sciatica: a systematic review and meta-analysis. Ann Intern Med 2012;157:865-77.

14. Chang-Chien GC, Knezevic NN, McCormick Z, et al. Transforaminal versus interlaminar approaches to epidural steroid injections: a systematic review of comparative studies for lumbosacral radicular pain. Pain Physician 2014;17:E509-24.

15. Staal JB, de Bie R, de Vet HCW, et al. Injection therapy for subacute and chronic low-back pain. Cochrane Database Syst Rev 2008;96:CD001824.

16. Bhatia A, Flamer D, Shah PS, et al. Transforaminal epidural steroid injections for treating lumbosacral radicular pain from herniated intervertebral discs: a systematic review and meta-analysis. Anesth Analg 2016;122:857-70.

17. Riew KD, Yin Y, Gilula L, et al. The effect of nerve-root injections on the need for operative treatment of lumbar radicular pain. $A$ prospective, randomized, controlled, double-blind study. J Bone Joint Surg Am 2000;82-A:1589-93.

18. Karppinen J, Malmivaara A, Kurunlahti M, et al. Periradicular infiltration for sciatica: a randomized controlled trial. Spine 2001;26:1059-67.

19. Tafazal S, Ng L, Chaudhary $\mathrm{N}$, et al. Corticosteroids in periradicular infiltration for radicular pain: a randomised double blind controlled trial. One year results and subgroup analysis. Eur Spine $J$ 2009;18:1220-5.

20. Ghahreman A, Ferch R, Bogduk N. The efficacy of transforaminal injection of steroids for the treatment of lumbar radicular pain. Pain Med 2010;11:1149-68.

21. Cohen SP, White RL, Kurihara C, et al. Epidural steroids, etanercept, or saline in subacute sciatica: a multicenter, randomized trial. Ann Intern Med 2012;156:551-9.

22. Manchikanti L, Cash KA, Pampati V, et al. Transforaminal epidural injections in chronic lumbar disc herniation: a randomized, doubleblind, active-control trial. Pain Physician 2014;17:E489-501.

23. Epstein NE. The risks of epidural and transforaminal steroid injections in the spine: commentary and a comprehensive review of the literature. Surg Neurol Int 2013;4(Suppl 2):74-93.

24. Manchikanti L, Candido KD, Singh V, et al. Epidural steroid warning controversy still dogging FDA. Pain Physician 2014;17:E451-74.

25. Rathmell JP, Benzon HT, Dreyfuss P, et al. Safeguards to prevent neurologic complications after epidural steroid injections: consensus opinions from a multidisciplinary working group and national organizations. Anesthesiology 2015;122:974-84.

26. El-Yahchouchi C, Geske JR, Carter RE, et al. The noninferiority of the nonparticulate steroid dexamethasone vs the particulate steroids betamethasone and triamcinolone in lumbar transforaminal epidural steroid injections. Pain Med 2013;14:1650-7.

27. Kennedy DJ, Plastaras C, Casey E, et al. Comparative effectiveness of lumbar transforaminal epidural steroid injections with particulate versus nonparticulate corticosteroids for lumbar radicular pain due to intervertebral disc herniation: a prospective, randomized, doubleblind trial. Pain Med 2014;15:548-55.

28. Denis I, Claveau G, Filiatrault M, et al. Randomized double-blind controlled trial comparing the effectiveness of lumbar transforaminal epidural injections of particulate and nonparticulate corticosteroids for lumbosacral radicular pain. Pain Med 2015;16:1697-708.

29. Roncoroni C, Baillet A, Durand M, et al. Efficacy and tolerance of systemic steroids in sciatica: a systematic review and meta-analysis. Rheumatology 2011;50:1603-11.

30. Balakrishnamoorthy R, Horgan I, Perez S, et al. Does a single dose of intravenous dexamethasone reduce symptoms in emergency department patients with low back pain and radiculopathy (SEBRA)? A double-blind randomised controlled trial. Emerg Med J 2015;32:525-30.

31. Goldberg H, Firtch W, Tyburski M, et al. Oral steroids for acute radiculopathy due to a herniated lumbar disk: a randomized clinical trial. JAMA 2015;313:1915-23.

32. Chou R, Hashimoto R, Friedly J, et al. Epidural corticosteroid injections for radiculopathy and spinal stenosis: a systematic review and meta-analysis. Ann Intern Med 2015;163:373-81. 
33. Chiarotto A, Deyo RA, Terwee CB, et al. Core outcome domains for clinical trials in non-specific low back pain. Eur Spine $J$ 2015;24:1127-42.

34. Fairbank JC, Pynsent PB. The Oswestry Disability Index. Spine 2000;25:2940-53.

35. Guzman JZ, Cutler HS, Connolly J, et al. Patient-reported outcome instruments in spine surgery. Spine 2016;41:429-37.

36. Williamson A, Hoggart B. Pain: a review of three commonly used pain rating scales. J Clin Nurs 2005;14:798-804.

37. Shaygan M, Böger A, Kröner-Herwig B. Clinical features of chronic pain with neuropathic characteristics: a symptom-based assessment using the pain DETECT questionnaire. Eur J Pain 2013;17:

38. Ware J. SF-36 Health survey: manual and interpretation guide. Boston: The Health Institute, New England Medical Cente, 1993.

39. Fardon DF, Milette PC. Combined Task Forces of the North American Spine Society, American Society of Spine Radiology, and American Society of Neuroradiology. Nomenclature and classification of lumbar disc pathology. Recommendations of the combined task forces of the North American Spine Society, American Society of Spine Radiology, and American Society of Neuroradiology. Spine 2001;26:E93-E113.

40. Pfirrmann CW, Dora C, Schmid MR, et al. MR image-based grading of lumbar nerve root compromise due to disk herniation: reliability study with surgical correlation. Radiology 2004;230:583-8.

41. Husereau D, Drummond M, Petrou S, et al. Consolidated health economic evaluation reporting standards (CHEERS) statement. BMJ 2013;346:f1049.

42. Dolan P. Modeling valuations for EuroQol health states. Med Care 1997:35:1095-108.

43. Commonwealth of Australia as represented by the Department of Health. Guidelines for preparing a submission to the Pharmaceutical
Benefits Advisory Committee (Version 5.0). 2016 https:// pbacpbsgovau/content/information/files/pbac-guidelines-version-5pdf

44. Manual of resource items and their associated unit costs. For use in submissions to the Pharmaceutical Benefits Advisory Committee involving economic analyses Version 5.0. 2016.

45. Koopmanschap MA. PRODISQ: a modular questionnaire on productivity and disease for economic evaluation studies. Expert Rev Pharmacoecon Outcomes Res 2005;5:23-8.

46. Leggett LE, Khadaroo RG, Holroyd-Leduc J, et al. Measuring resource utilization: a systematic review of validated self-reported questionnaires. Medicine 2016;95:e2759.

47. Lauridsen HH, Hartvigsen J, Manniche C, et al. Responsiveness and minimal clinically important difference for pain and disability instruments in low back pain patients. BMC Musculoskelet Disord 2006;7:82.

48. Ostelo RW, Deyo RA, Stratford P, et al. Interpreting change scores for pain and functional status in low back pain: towards international consensus regarding minimal important change. Spine 2008;33:90-4.

49. Proschan MA, Waclawiw MA. Practical guidelines for multiplicity adjustment in clinical trials. Control Clin Trials 2000;21:527-39.

50. Wason JM, Stecher L, Mander AP. Correcting for multiple-testing in multi-arm trials: is it necessary and is it done? Trials 2014;15:364.

51. Bender R, Lange S. Adjusting for multiple testing - when and how? $J$ Clin Epidemiol 2001;54:343-9.

52. CONSORT. Welcome to the CONSORT website. $2010 \mathrm{http} / / / \mathrm{www}$. consort-statement.org/

53. White IR, Horton NJ, Carpenter J, et al. Strategy for intention to treat analysis in randomised trials with missing outcome data. BMJ 2011;342:d40. 